\section{INTRODUCCIÓN}

Las infecciones del tracto urinario (ITUs) son una de las enfermedades más prevalentes en el mundo, y consisten en la colonización y multiplicación microbiana, habitualmente bacteriana. Los datos epidemiológicos demuestran una mayor incidencia de ITUs en la población con diabetes meIlitus (DM) tipo 1 y 2 , siendo más prevalente con la edad, género femenino, mal control glucémico y deterioro de la función renal. Las ITUs pueden clasificarse por el nivel anatómico, su grado de severidad y los factores de riesgo para una infección.

Ante la situación de mal control metabólico, hiperglucemia/hipoglucemia o estados hiperosmolares o cetoacidosis es necesario realizar un diagnóstico temprano de posible ITU, por ende su reconocimiento precoz y adecuado manejo son importantes.
Las características clínicas y los datos de laboratorio se centran en el nivel anatómico y el grado de gravedad de la infección. En la evaluación clínica de rutina, antes de establecer un diagnóstico, debe considerarse una serie de criterios básicos que incluyen:

- Síntomas clínicos.

- Resultados de pruebas de laboratorio.

- Evidencia de la presencia de microorganismos mediante cultivos u otras pruebas específicas.

Debido al crecimiento sostenido de la DM a nivel mundial -y por ende la enfermedad renal por diabetes como causa principal de insuficiencia renal crónica (IRC)- será cada vez más habitual tratar pacientes con ambas patologías, grupo que presenta mayor susceptibilidad a las infecciones, y principalmente a las del tracto urinario.

\title{
INFECCIONES DEL TRACTO URINARIO EN PACIENTES CON DIABETES. GENERALIDADES
}

\section{URINARY TRACT INFECTIONS IN PATIENTS WITH DIABETES. OVERVIEW}

\author{
Jimena Soutelo ${ }^{1}$
}

\section{RESUMEN}

La infección del tracto urinario (ITU) reside en la colonización y mutiplicación de microorganismos en el tracto urinario. Se diagnostica por cultivo bacteriano y se clasifica según la localización anatómica y la severidad que determinan los signos, síntomas y gravedad de la infección, recurrencia y factores de riesgo. Las ITUs más frecuentemente adquiridas ocurren en la comunidad. Existe mayor incidencia de este tipo de infecciones en las personas con diabetes mellitus, siendo más prevalente con la edad, género femenino, mal control glucémico y pobre función renal.

Palabras clave: infección del tracto urinario; diabetes mellitus; clasificación anatómica; grados de severidad.

Revista de la Sociedad Argentina de Diabetes 2019; Vol. 53 (28-50)

\begin{abstract}
The urinary tract infection (UTI) lies in the colonization and multiplication of microorganisms in the urinary tract. It is diagnosed by bacterial culture and classified according to the anatomical location and the severity determining the signs, symptoms and severity of the infection, recurrence and risk factors. The most frequently acquired UTIs occur in the community. There is a higher incidence of this type of infection in people with diabetes mellitus, being more prevalent with age, female gender, poor glycemic control and poor renal function.
\end{abstract}

Key words: urinary tract infection; diabetes mellitus; anatomical classification; severity scale.

Revista de la Sociedad Argentina de Diabetes 2019; Vol. 53 (28-50)
Médica especialista en Endocrinología, Servicio de Endocrinología, Hospital Churruca Visca, Jefa de Trabajos Prácticos del Departamento de Fisiología, Unidad Académica 2, Universidad de Buenos Aires, Ciudad Autónoma de Buenos Aires, Argentina
Contacto de la autora: Jimena Soutelo

E-mail:mjimenasoutelo@gmail.com

Correspondencia: Uspallata 3400 (C1437JCP), Ciudad Autónoma de Buenos Aires, Argentina

Fecha de trabajo recibido: 25/03/19

Fecha de trabajo aceptado: 03/04/19

Conflicto de interés: la autora declara que no existe conflicto de interés 


\section{Definición}

La infección del tracto urinario (ITU) consiste en la colonización y multiplicación microbiana, habitualmente bacteriana, a lo largo del trayecto del tracto urinario. Se denomina pielonefritis si afecta al riñón y la pelvis renal, cistitis si implica la vejiga, uretritis si afecta a la uretra y prostatitis si se localiza en la próstata.

Podemos definir ITU con los siguientes conteos bacterianos:

- $\geq 10^{3} \mathrm{UFC} / \mathrm{ml}$ (unidad formadora de colonias/ml) de uropatógenos en una muestra de orina tomada de la mitad del chorro en mujeres con síntomas de cistitis aguda no complicada.

- $\geq 10^{4} \mathrm{UFC} / \mathrm{ml}$ de uropatógenos en una muestra de orina tomada de la mitad del chorro en mujeres con síntomas de pielonefritis aguda no complicada.

- $\geq 10^{5} \mathrm{UFC} / \mathrm{ml}$ de uropatógenos en una muestra de orina tomada de la mitad del chorro en mujeres ó $\geq 10^{4} \mathrm{UFC} / \mathrm{ml}$ de uropatógenos en una muestra de orina tomada de la mitad del chorro en hombres, o en la orina de catéter en mujeres con una infección urinaria complicada'.

\section{Clasificación}

Las ITUs pueden clasificarse por el nivel anatómico, su grado de severidad y los factores de riesgo para una infección. Así, los signos, síntomas y laboratorio se centran en el nivel anatómico y el grado de gravedad de la infección, mientras que los factores de riesgo (Tablas 1 y 2) contribuyen a definir cualquier medida terapéutica adicional requerida.

- Según categorías anatómicas: infecciones bajas (uretritis y cistitis) e infecciones altas (pielonefritis aguda, abscesos intrarrenales y perinéfricos). Pueden presentarse juntas o independientes, pudiendo ser asintomáticas o formando parte de un síndrome clínico?.

- En función de su recurrencia: se define recurrencia esporádica a la presencia de $\leq 1$ ITUs/6 meses y/o $\leq 2$ ITUs/año, y recurrente a la presencia de $\geq 2$ ITUs/6 meses y/o $\geq 3$ ITUs/año. La recurrencia puede deberse a una recidiva o una reinfección. La infección que recidiva luego de la administración de antibióticos se debe a la persistencia de la cepa infectante original, mientras que la reinfección a la presencia de una nueva cepa'. Asimismo existen otras formas de clasificación de ITUs (Gráfico 1)².

\section{Epidemiología}

Según información proveniente de Estados Unidos, las ITUS contraídas en la comunidad son muy comunes y suponen más de 7 millones de consultas médicas anuales, con una incidencia de 0,5 a 0,7 infecciones/paciente/año en mujeres jóvenes. Las ITUS sintomáticas son infrecuentes en hombres de $<50$ años. Después de los 65 años, en personas no institucionalizadas, la tasa de ITUs es del 10,9\% para hombres y $14 \%$ para mujeres ${ }^{3}$. Algunos autores reportaron una prevalencia de ITUs del 9-20\% entre las mujeres y $3-11 \%$ en los hombres con diabetes ${ }^{4}$. Wilke et al. hallaron en una población alemana de 456.586 pacientes con diabetes tipo 2 (DM2) una incidencia de infección urinaria de 87,3 eventos/1.000 pacientes/año, y observaron una fuerte asociación de ITUs con la edad, género femenino, mal control metabólico $(\mathrm{HbA} 1 \mathrm{c}>9,5 \%)$, grado de función renal ( $F G<60 \mathrm{ml} / \mathrm{min})$, comorbilidades y uso de insulina ${ }^{5}$. En un estudio holandés realizado entre 1995-2003 en pacientes con DM2 se detectó una incidencia de ITU de 72-101 eventos/1.000 pacientes/año. Asimismo en otro estudio realizado en el Reino Unido entre 1990-2008 en una muestra de pacientes con DM2 se observó una incidencia de 46,9 eventos/1.000 pacientes/ año ${ }^{5}$. Todos los autores coinciden en una asociación de ITU con mal control glucémico y pobre función renal. Recientemente Nichols et al. estudiaron una población de 78.590 individuos con y sin diabetes y descubrieron que los pacientes con diabetes presentaban una incidencia de ITU de 68,5 eventos/1.000 persona/año en EE.UU., versus una incidencia de 54,8 eventos/1.000 personas/ año en los individuos sin diabetes. Además observaron mayor tasa de infecciones genitales en la población con diabetes, y una mayor prevalencia de ambas infecciones en el género femenino con y sin diabetes ${ }^{6}$. En el estudio Diabetes Control and Complications Trial/Epidemiology of Diabetes Interventions and Complications (DCCT/ EDIC) la prevalencia de ITU en mujeres con DM1 fue del $15 \%$ anual según el control glucémico. Por cada aumento de una unidad en la $\mathrm{HbA} 1 \mathrm{c}$ se evidenció un aumento del $21 \%$ en el número de ITUs. Esta asociación fue independiente de los factores de riesgo conocidos de infección como raza, histerectomía, ITU previa, actividad sexual, nefropatía, neuropatía periférica y autónoma. 
Esto sugiere que la glucemia en sí misma puede afectar de forma independiente la frecuencia de ITU en pacientes con diabetes?

En conclusión, los datos epidemiológicos de- muestran una mayor incidencia de infecciones del tracto urinario en la población con DM1 y DM2, siendo más prevalente con la edad, género femenino, mal control glucémico y pobre función renal.

\begin{tabular}{|c|c|c|}
\hline Tipo & Categoría de factor de riesgo (FR) & Ejemplo de FR \\
\hline $\mathbf{O}$ & FR no conocido o no asociado & Mujeres premenopáusicas sanas \\
\hline $\mathbf{R}$ & $\begin{array}{l}\text { FR de ITU recurrente } \\
\text { Sin riesgo de severidad }\end{array}$ & $\begin{array}{l}\text { Comportamiento sexual y dispositivos. Contraconceptivos } \\
\text { Deficiencia hormonal (mujer postmenopáusica) } \\
\text { Diabetes mellitus controlada }\end{array}$ \\
\hline $\mathbf{E}$ & $\begin{array}{l}\text { FR de ITU recurrente } \\
\text { Sin riesgo de severidad }\end{array}$ & $\begin{array}{l}\text { Embarazo } \\
\text { Género masculino } \\
\text { Diabetes mellitus mal controlada } \\
\text { Inmunosupresión } \\
\text { Enfermedades del tejido conectivo }\end{array}$ \\
\hline $\mathbf{N}$ & $\begin{array}{l}\text { Enfermedad nefrológica } \\
\text { Con mayor riesgo de resultados graves }\end{array}$ & $\begin{array}{l}\text { Insuficiencia renal } \\
\text { Poliquistosis renal }\end{array}$ \\
\hline U & $\begin{array}{l}\text { FR urológico } \\
\text { Con riesgo de resultado grave } \\
\text { Que puede resolverse con la terapia }\end{array}$ & $\begin{array}{l}\text { Obstrucción ureteral } \\
\text { Catéter transitorio del tracto urinario } \\
\text { Bacteriuria asintomática con otro FR } \\
\text { Vejiga neurogénica controlada } \\
\text { Cirugía urológica }\end{array}$ \\
\hline C & $\begin{array}{l}\text { Catéter urinario permanente } \\
\text { Con FR urológico } \\
\text { Con riesgo de un resultado grave }\end{array}$ & $\begin{array}{l}\text { Tratamiento de catéter de vías urinarias } \\
\text { Obstrucción urinaria } \\
\text { Disfunción vesical }\end{array}$ \\
\hline
\end{tabular}

Tabla 1: Evaluación del factor de riesgo según ORENUC.

\begin{tabular}{|l|l|}
\hline \multicolumn{1}{|c|}{ Mujeres premenopáusicas } & \multicolumn{1}{c|}{ Mujeres postmenopáusicas } \\
\hline Relaciones sexuales & Historia de ITU antes de la menopausia \\
Uso de espermicida & Incontinencia urinaria \\
Nueva pareja sexual & Vaginitis atrófica por deficiencia de estrógenos \\
Madre con historial de ITU & Cistocele \\
Historia de ITU durante la infancia & Aumento del volumen residual \\
& $\begin{array}{l}\text { Estado de secreción del antígeno del grupo sanguíneo } \\
\text { Colocación de catéter vesical y deterioro funcional } \\
\text { Institucionalización en edad avanzada }\end{array}$ \\
\hline
\end{tabular}

Tabla 2: Factores de riesgo de ITU relacionados con la edad en la mujer.

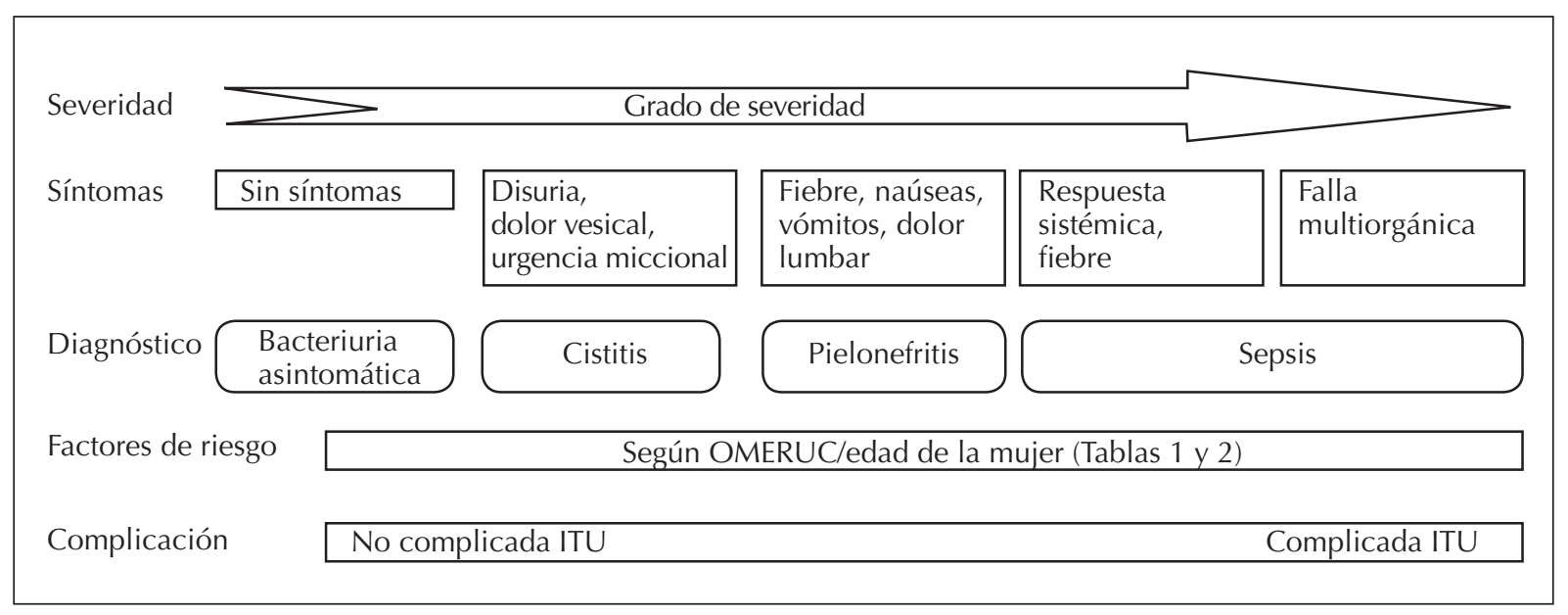

Adaptado de: Bjerklund-Johansen et al.

Gráfico 1: Visión sinóptica de la clasificación de la ITU. 


\section{BIBLIOGRAFÍA}

1. Grabe M, Bartoletti R, Bjerklund Johansen TE, et al. Guidelines on urological infections. European Association of Urology 2015. Disponible en: http://www.uroweb.org/guidelines/onlineguidelines/.

2. Bjerklund-Johansen TE, Botto $H$, Cek $M$, et al. Critical review of current definitions of urinary tract infections and proposal of an EAU/ESIU classification system. Int J Antimicrob Agents 2011; 38 Suppl S: 64-70. Disponible en: http://www.ncbi.nlm. nih.gov/pubmed/22018988.

3. Tandogdu Z, Wagenlehner FM. Global epidemiology of urinary tract infections. Curr Opin Infect Dis 2016; 29(1):73-9.

4. Chiu PF, Wu CL, Huang $\mathrm{CH}$, et al. Lower blood glucose and variability are associated with earlier recovery from renal injury caused by episodic urinary tract infection in advanced type 2 diabetic chronic kidney disease. PLoS ONE 2014; 26 9(9): e108531. Doi:10.1371/journal.pone.0108531.
5. WilkeT, Boettger B, Berg B, et al. Epidemiology of urinary tract infections in type 2 diabetes mellitus patients: an analysis based on a large sample of 456,586 GermanT2DM patients. J Diabetes Complications 2015; 29(8): 1015-23.

6. Nichols GA, Brodovicz KG, Kimes TM, et al. Prevalence and incidence of urinary tract and genital infections among patients with and without type 2 diabetes. J Diabetes Complications 2017; 31(11): 1587-91.

7. Lenherr SM, Clemens JQ, Braffett BH, Cleary PA, Dunn RL, Hotaling JM, Jacobson AM, Kim C, Herman W, Brown JS, Wessells H, Sarma AV; The DCCT/EDIC Research Group. Glycemic control and urinary tract infections in women with type 1 diabetes: results from the DCCT/EDIC. J Urol 2016; 196(4): 1129-35.

\section{PATOGENIA. FACTORES DE RIESGO EN DIABETES MELITUS}

\section{PATHOGENESIS. RISK FACTORS IN DIABETES MELLITUS}

Teresa Bensusan ${ }^{1}$

\section{RESUMEN}

La interacción entre uroepitelio y uropatógeno, base de la patogenia de las infecciones del tracto urinario (ITUs), puede derivar en la eliminación bacteriana por parte de la célula huésped o la invasión y multiplicación bacteriana. Dentro de la célula huésped los uropatógenos pueden perturbar las defensas y resistir el tratamiento antibiótico. En pacientes con diabetes, especialmente con enfermedad renal por diabetes, se ha demostrado una reducción de la capacidad de inhibición de la adherencia bacteriana al uroepitelio, por ende mayor posibilidad de invasión bacteriana. La glicosilación de todos los elementos del sistema inmune, incluida la menor liberación de factores como las interleuquinas a nivel urinario y la alteración del vaciamiento vesical por neuropatía autonómica, favorecen el desarrollo de este tipo de infecciones.

Palabras clave: uroepitelio; uropatógeno; glicoproteína de Tamm Horsfall; glicosilación; uroplaquinas.

Revista de la Sociedad Argentina de Diabetes 2019; Vol. 53 (28-50)

\section{ABSTRACT}

Interaction between urothelium and uropathogen, the basis of the pathogenesis of urinary tract infections (UTIS), can lead to bacterial elimination by the host cell or bacterial invasion and multiplication. Inside the host cell, uropathogens can impair defenses and resist antibiotic treatment. In patients with diabetes, especially diabetes-related kidney disease, a reduction in the inhibition capacity of bacterial adherence to the urothelium has been demonstrated; therefore, a highest chance of bacterial invasion. The glycosylation of all elements of the immune system, including the lower release of factors such as interleukins at the urinary level and the impairment of bladder emptying by autonomic neuropathy, enhance the development of this type of infections.

Key words: urothelium; uropathogen; Tamm Horsfall glycoprotein; glycosylation; uroplakins.

Revista de la Sociedad Argentina de Diabetes 2019; Vol. 53 (28-50)
' Médica especialista en Clínica Médica y Diabetes, Centro Médico Cepem, Morón, Provincia de Buenos Aires, Argentina
Contacto de la autora: Teresa Bensusan

E-mail: tbensusan@gmail.com

Correspondencia: República Oriental del Uruguay 387

(CP1780), Morón, Provincia de Buenos Aires, Argentina

Fecha de trabajo recibido: 25/03/19

Fecha de trabajo aceptado: 03/04/19

Conflicto de interés: la autora declara que no existe conflicto de interés. 